\title{
Cinobufagin inhibits tumor growth by inducing apoptosis through Notch signaling pathways in human cholangiocarcinoma
}

\author{
Jiajun Ren ${ }^{1}$, Shouhua Wang ${ }^{2}$, Longyang Jin ${ }^{2}$, Fei Ma ${ }^{3}$, Di Zhou ${ }^{2}$, Qiang Cai ${ }^{1}$ \\ ${ }^{1}$ Department of General Surgery, Shanghai Institute of Digestive Surgery, Ruijin Hospital, Shanghai Jiaotong University School of Medicine, \\ Shanghai 200025, China; ${ }^{2}$ Department of General Surgery, ${ }^{3}$ Department of Oncology, Xinhua Hospital, Shanghai Jiaotong University School of \\ Medicine, Shanghai 200092, China \\ Contributions: (I) Conception and design: J Ren, D Zhou, Q Cai; (II) Administrative support: D Zhou, Q Cai; (III) Provision of study materials or \\ patients: S Wang, L Jin; (IV) Collection and assembly of data: S Wang, F Ma; (V) Data analysis and interpretation: L Jin, F Ma; (VI) Manuscript \\ writing: All authors; (VII) Final approval of manuscript: All authors. \\ Correspondence to: Qiang Cai. Department of General Surgery, Shanghai Institute of Digestive Surgery, Ruijin Hospital, Shanghai Jiaotong University \\ School of Medicine, 197 Ruijin No. 2 Road, Shanghai 200025, China. Email: cq01a35@rjh.com.cn; Di Zhou. Department of General Surgery, Xinhua \\ Hospital, Shanghai Jiaotong University School of Medicine, 1665 Kongjiang Road, Shanghai 200092, China. Email: zhoudi@xinhuamed.com.cn.
}

\begin{abstract}
Background: Many studies have shown that cinobufagin has antitumor effects against many cancers. The aim of this study was to assess the effects of cinobufagin on cholangiocarcinoma (CCA) cells.

Methods: Colony formation assay, cell-counting kit-8 (CCK-8) assay, and tumor xenograft experiments were applied to investigate the function of cinobufagin on human CCA cells, in vitro and in vivo. Flow cytometric analysis was performed to validate the effects of cinobufagin on cell apoptosis. Quantitative realtime reverse transcription PCR (qRT-PCR) and Western blot assays were performed to evaluate expression levels of related genes.
\end{abstract}

Results: This study found that cinobufagin inhibited proliferation in both QBC939 and RBE cells. Flow cytometric analysis indicated that rate of apoptosis was significantly increased when treated with cinobufagin. Regarding mechanism analysis, cinobufagin was found to dramatically inactivate Notch signaling pathways. Animal experiment results in vivo were consistent with outcomes in vitro.

Conclusions: These data suggest that cinobufagin-mediated inactivation of Notch pathways may play an important role in the induction of apoptosis in CCA.

Keywords: Cholangiocarcinoma (CCA); cinobufagin; growth; apoptosis; Notch signaling

Submitted Feb 26, 2019. Accepted for publication Sep 30, 2019.

doi: $10.21037 /$ tcr.2019.10.06

View this article at: http://dx.doi.org/10.21037/tcr.2019.10.06

\section{Introduction}

Cholangiocarcinoma (CCA), the second most common primary liver malignancy, is an epithelial cell malignancy arising from varying locations in the biliary tree $(1,2)$. CCA is highly malignant and generally fatal with extremely poor survival rates (3). Owing to the lack of specific symptoms and signs, most CCA patients are diagnosed at advanced stage and missing the optimal time for operation (4). Although several new-targeted therapies have been developed, surgical resection remains the only potential curative treatment. Unfortunately, only a few tumors are resectable and curable at diagnosis and the 5 -year survival rate following resection is only about $20 \%$ (5). Furthermore, the epidemiological studies showed a global trend of rising morbidity and mortality of CCA over the past decade, especially in China (6). Thus, the development of novel and promising therapeutic approaches is an urgent task.

Chansu is a Traditional Chinese Medicine used in China for hundreds of years, extracted from parotid glands and skin glands of the Chinese toad (Bufo gargarizan) (7-9). In China and some Asian countries, it has been extensively used to treat a variety of diseases, including inflammation, 
Table 1 Primers for genes used in the study

\begin{tabular}{lll}
\hline Gene name & Forward primer & Reverse primer \\
\hline GAPDH & GGAGCGAGATCCCTCCAAAAT & GGCTGTTGTCATACTTCTCATGG \\
Notch1 & GAGGCGTGGCAGACTATGC & CTTGTACTCCGTCAGCGTGA \\
Hes-5 & TCAACACGACACCGGATAAAC & GCCGCGAGCTATCTTCTTCA \\
Hey-1 & GAAAACCGACTGCGGAAGC & GACGAAGGCTTTGCTGTGCT \\
\hline
\end{tabular}

CNS respiratory diseases, cardiac disease, and cancer. Cinobufagin is one of the major active components in Chansu, isolated and purified in the past few decades $(10,11)$. It has reportedly been used for anti-cancer actions including inhibition of prostate cancer cells by inducing apoptosis and migration proliferation of human hepatic cells $(12,13)$. Furthermore, cinobufagin could also restrain other cancer rapid progression, such as non-small cell lung cancer, breast cancer, osteosarcoma (14-16). Additionally, the toxicity of cinobufagin to normal cells is low and it is suitable as a therapeutic agent.

However, whether cinobufagin affects CCA remains unclear. This current study investigated the antitumor effects of cinobufagin on CCA. Results illustrated that cinobufagin exhibited significant suppression effects on CCA cell proliferation as well as inducing cellular apoptosis, both in vitro and in vivo. As is well-known, the Notch signaling pathway plays a vital role in the fundamental processes of cancer cell proliferation and apoptosis. In the present study, we found that cinobufagin may inhibit tumor growth by inducing apoptosis through the activation of Notch signaling pathways in CCA.

\section{Methods}

\section{Cell culture}

Human CCA cell lines QBC939 (extrahepatic) and RBE (intrahepatic) were provided by Department of General Surgery, Xinhua Hospital, Shanghai Jiaotong University School of Medicine, Shanghai, China $(17,18)$. QBC939 and RBE were cultured in DMEM containing 10\% fetal bovine serum (FBS) and $1 \%$ antibiotics (penicillin $100 \mathrm{U} / \mathrm{mL}$, streptomycin $100 \mu \mathrm{g} / \mathrm{mL}$ ). Cells were maintained at $37{ }^{\circ} \mathrm{C}$ with $5 \% \mathrm{CO}_{2}$. Cinobufagin was purchased from Chinese Materials Research Center (Beijing, China) and dissolved in dimethyl sulfoxide.

\section{Cell-counting kit-8 (CCK-8) and colony formation assays}

For CCK-8 assay, 1,000 cells were seeded and cultured in a 96-well plate. Viability of cells was detected with CCK8 assay, according to instructions, at indicated time points. $\mathrm{OD}_{450}$ values were proportional to the viability of cells. For colony formation assay, 1,000 cells were seeded and cultured in 6-well plate. The colony number was counted after 14 days of culturing. All experiments were performed in triplicate.

\section{Flow cytometric apoptosis assay}

After respective treatment, cells were collected and washed with PBS. Next, apoptotic cell death rates were examined by Annexin V-FITC and PI double staining using the Annexin V-FITC Apoptosis Detection Kit, according to manufacturer instructions.

\section{Quantitative real-time PCR (qPCR)}

Total RNA was extracted with TRIzol Reagent. Reverse transcription was performed using a TOYOBO Reverse Transcription Kit. qPCR systems were set up in triplicate and run on the 7900 PCR machine with SYBR Green PCR Master Mix. Relative gene expression was calculated using the $2^{-\triangle \Delta C T}$ method and normalized to GAPDH. Primers used are listed in Table 1.

\section{Western blot}

Protein was extracted with RIPA buffer. Protein lysates were then separated by electrophoresis on $10 \%$ sodium dodecyl sulfate polyacrylamide gel and transferred to PVDF membranes. Membranes were then blocked in 5\% skim milk for 2 hours. They were then incubated with primary antibodies overnight at $4{ }^{\circ} \mathrm{C}$. After 2 hours of 

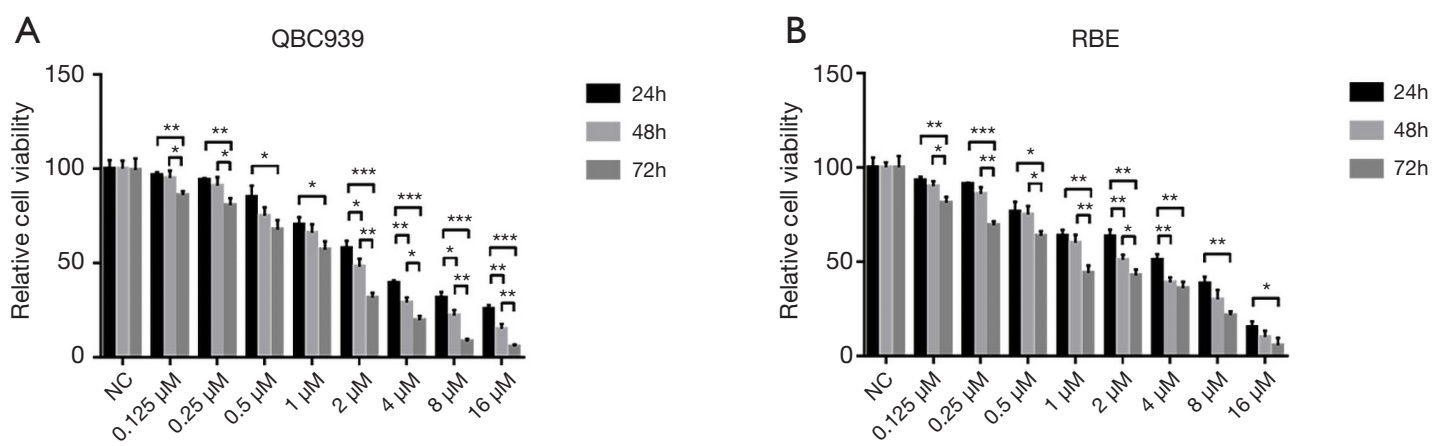

Figure 1 Cinobufagin inhibits cell proliferation of human CCA. (A) CCK-8 assay in cell line QBC939 treated with various concentrations of cinobufagin for 24, 48 and $72 \mathrm{~h}$ is shown; (B) CCK-8 assay in cell line RBE treated with various concentrations of cinobufagin for 24,48 and $72 \mathrm{~h}$ is shown. Mean $\pm \mathrm{SD}$ of triplicate experiments were plotted, ${ }^{*} \mathrm{P}<0.05,{ }^{* *} \mathrm{P}<0.01,{ }^{* * *} \mathrm{P}<0.001$. CCA, cholangiocarcinoma; CCK-8, cell-counting kit-8.

incubation with secondary antibodies at $37^{\circ} \mathrm{C}$, membranes were detected with enhanced chemiluminescence. Primary antibody of NICD1 was purchased from Abcam and GAPDH was from ProteinTech. Caspase-3, cleaved Caspase- 3 and Bax were obtained from Cell Signaling Technology.

\section{Xenograft study}

The xenograft study was approved by the Experimental Animal Ethics Committee of Ruijin Hospital and carried out according to the Guide for the Institutional Animal Care and Use Committee (IACUC) of Shanghai Jiaotong University. Ten four-week-old nude mice were subcutaneously injected with $5 \times 10^{6}$ QBC939 cells. Two weeks after injection, the mice were randomized into 2 groups ( $\mathrm{n}=5$ mice/group) for the following treatments: vehicle control and cinobufagin $(10 \mathrm{mg} / \mathrm{kg}$, i.p. injection, twice a week). Tumor sizes and body weight were monitored and measured every 3 days. After 2 weeks of treatment, the mice were euthanized and tumor tissue was collected.

\section{Statistical analysis}

Statistical analyses were performed with SPSS16.0. Data are presented as mean \pm standard deviation. Student's $t$-test or one-way ANOVA were used for statistical analysis when appropriate. $\mathrm{P}$ values $<0.05$ were considered statistically significant.

\section{Results}

\section{Cinobufagin inbibited CCA cell proliferation in vitro}

As one previous study has demonstrated, cinobufagin has the ability to suppress cancer cell growth in several tumors. This present study investigated the cytostatic and cytotoxic effects of cinobufagin in two human CCA cell lines. As shown in Figure 1A, CCK-8 assay indicated that proliferation of QBC939 cells was significantly inhibited by cinobufagin in a dose-dependent and time-dependent manner. A similar effect was also observed in RBE cells (Figure $1 B$ ). And the half-maximal inhibitory concentration of cinobufagin at $48 \mathrm{~h}$ was 2.08 and $1.93 \mu \mathrm{M}$ in QBC939 and RBE cells, respectively. Then we established an optimum concentration of cinobufagin in $2 \mu \mathrm{M}$ for the subsequent experiments. To further validate the role of cinobufagin, colony formation assay was conducted with the same phenomenon observed. The colony formation ability of QBC939 and RBE cells was dramatically suppressed by cinobufagin (Figure $2 A, B, C, D$ ). Taken together, these results suggest that cinobufagin could inhibit CCA cell growth in vitro.

\section{Cinobufagin induced apoptosis of human CCA cells}

Cell apoptosis is one of the major causes of cell growth inhibition. It has been reported that cinobufagin can induce apoptosis in osteosarcoma cells. To determine whether cell apoptosis contributes to cinobufagin-induced cell growth 


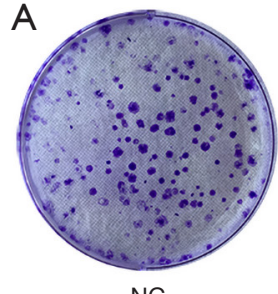

NC

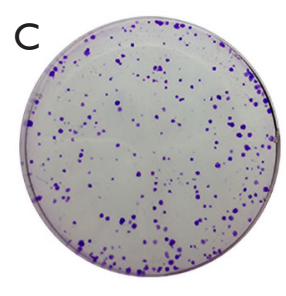

NC

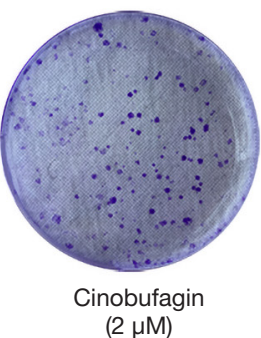

QBC939

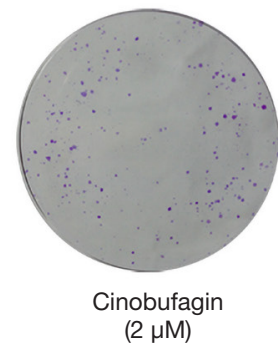

B
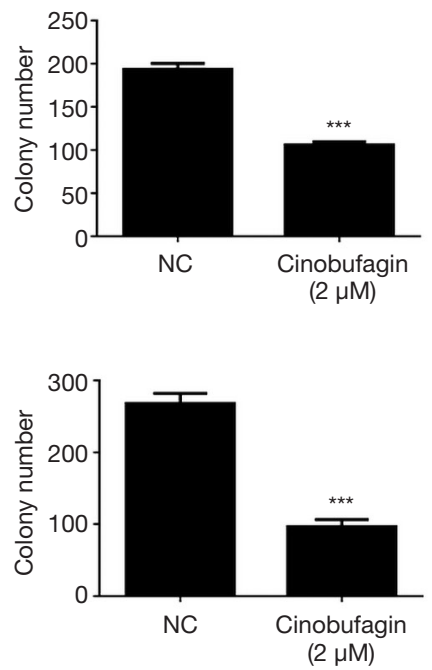

Figure 2 Cinobufagin inhibits colony formation of human CCA. (A,B) Cell colony formation assay in human CCA cell line QBC939 treated with cinobufagin is shown; (C,D) cell colony formation assay in human CCA cell line RBE treated with cinobufagin is shown. Mean \pm SD of triplicate experiments were plotted, ${ }^{* * *} \mathrm{P}<0.001$. CCA, cholangiocarcinoma.

inhibition in CCA cells, this study assessed apoptosis change during treatment with cinobufagin by flow cytometry with propidium iodide (PI) staining. Compared to controls, the percentage of apoptotic cells increased from $5.6 \%$ to $16.2 \%$ and $4.9 \%$ to $14.8 \%$ in QBC939 and RBE cells, respectively, after 24 hours of treatment with cinobufagin (Figure $3 A, B, C, D)$. Next, expression of apoptosis-related proteins was tested. As shown in Figure 3E,F, expression of apoptosis-promoting genes Caspase 3, cleaved Caspase3 and Bax was significantly induced by cinobufagin treatment. Therefore, this data suggests that cinobufagin may inhibit growth of CCA cells by inducing apoptosis.

\section{Cinobufagin inactivated Notch signaling pathways in buman CCA cells}

To further study the underlying mechanisms of cinobufagininduced inhibition, cell survival Notch pathways were examined. Notch signaling pathways are involved in the development and progression of several malignancies, including CCA. This study assessed expression of Notch-1 and its target genes, Hes-1, Hes-5, and Hey-1, in CCA cells using qPCR. Results indicated that cinobufagin significantly inhibited expression of Notch signaling pathway elements both in QBC939 and RBE cells (Figure 4A,B). Interaction of Notch ligands with their receptors promotes the release of Notch1 intracellular domain (NICD1), resulting in activation of the pathway. NICD1 translocates to the nucleus and induces target genes like Hes-1, Hes-5, and Hey-1. Next, expression of NICD1 was examined. As Figure $4 C, D$ show, cinobufagin obviously decreased protein expression of NICD1 in two CCA cell lines. These results suggest that cinobufagin may inhibit growth and induce apoptosis of CCA cells through Notch signaling pathways.

\section{Cinobufagin attenuated growth of CCA in vivo}

To examine the anti-tumor effects of cinobufagin, in vivo, nude mice were injected with QBC939 cells. After treatment with cinobufagin for 2 weeks, the mice were euthanized and tumor tissue was collected. During treatment, tumor growth curves exhibited significant differences, suggesting that cinobufagin inhibits tumor growth according to time (Figure 5A). Overall, tumors in the cinobufagin group were significantly smaller than those in the control group (Figure 5B). Furthermore, we monitored the body weight growth curve of nude mice after cinobufagin treatment, and the result showed that the side effect of cinobufagin is minimal in vivo (Figure S1). Moreover, expression of Caspase 3, cleaved Caspase3 and Bax in tumors was significantly induced while NICD1 was dramatically inhibited by cinobufagin treatment (Figure 5C). Thus, 
A

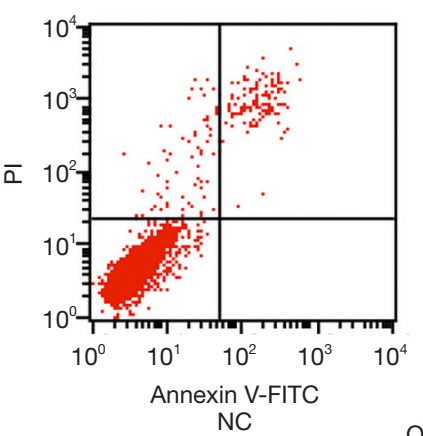

$\mathrm{NC}$

C

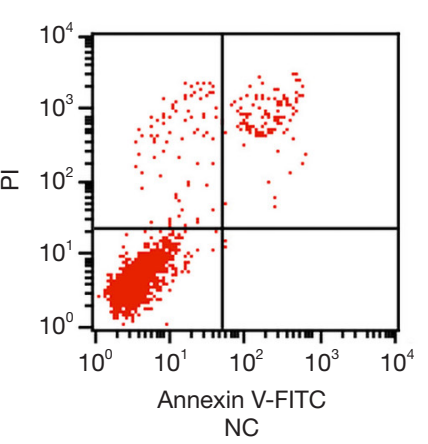

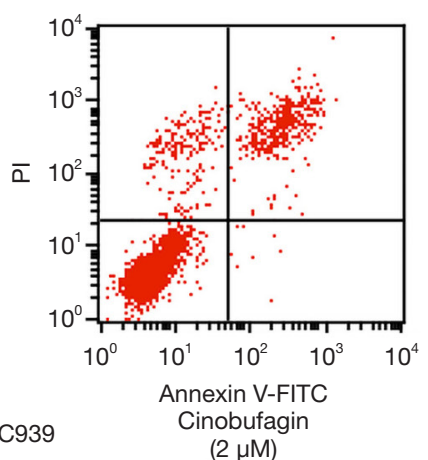

QBC939

$(2 \mu \mathrm{M})$

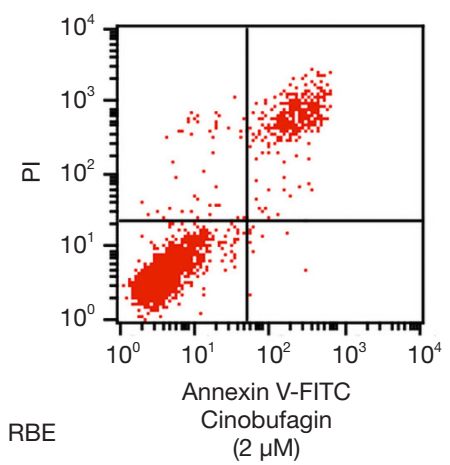

B

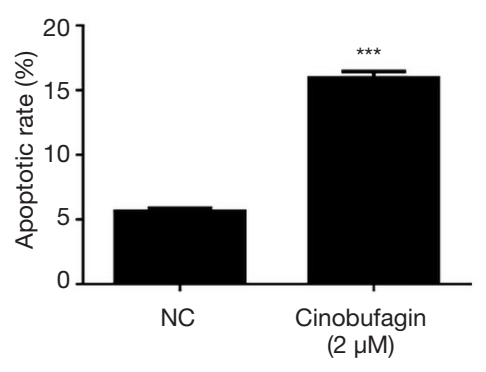

D

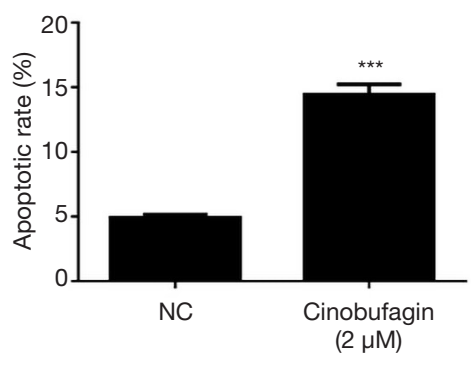

$E$

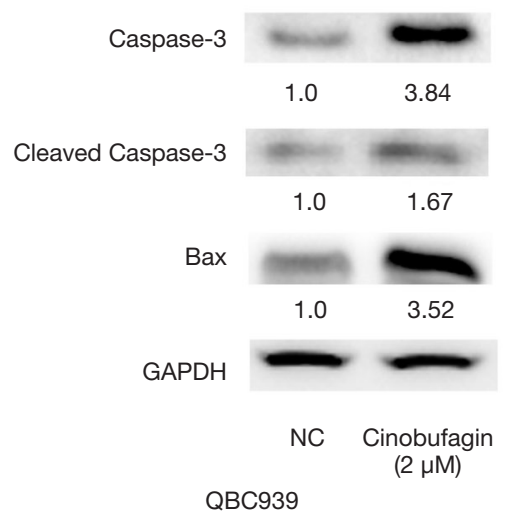

$\mathrm{F}$

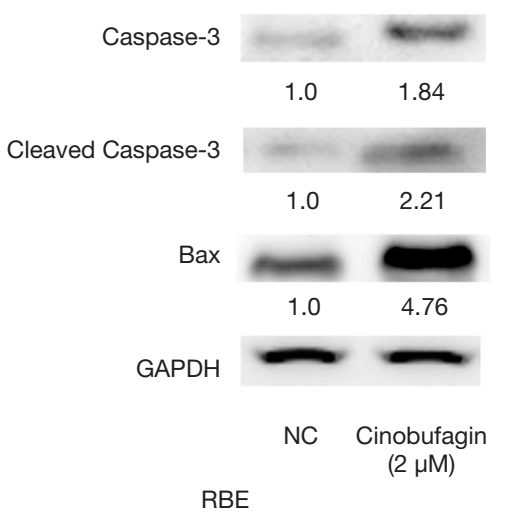

Figure 3 Cinobufagin induces apoptosis in human CCA cells. (A,B) Flow cytometric analysis for apoptosis in human CCA cell line QBC939 treated with cinobufagin is shown; (C,D) flow cytometric analysis for apoptosis in human CCA cell line RBE treated with cinobufagin is shown; (E,F) protein levels of Caspase-3 and Bax in cell lines QBC939 and RBE treated with cinobufagin were detected by Western blot. Mean \pm SD of triplicate experiments were plotted, ${ }^{* * *} \mathrm{P}<0.001$. CCA, cholangiocarcinoma.

cinobufagin could attenuate tumor growth by inducing apoptosis through Notch signaling pathways in a xenograft model of CCA.

\section{Discussion}

Incidence and mortality of CCA have been increasing in recent years (19-21). Surgery is still the most effective treatment option. CCA is typically diagnosed at an advanced stage, making surgical treatment ineffective. Development of a novel helpful treatment is an urgent task. Cinobufagin is an important cardenolidal steroid, extracted from skin secretions of the Chinese giant toad. It has been reported that cinobufagin had antitumor effects in several tumors, 

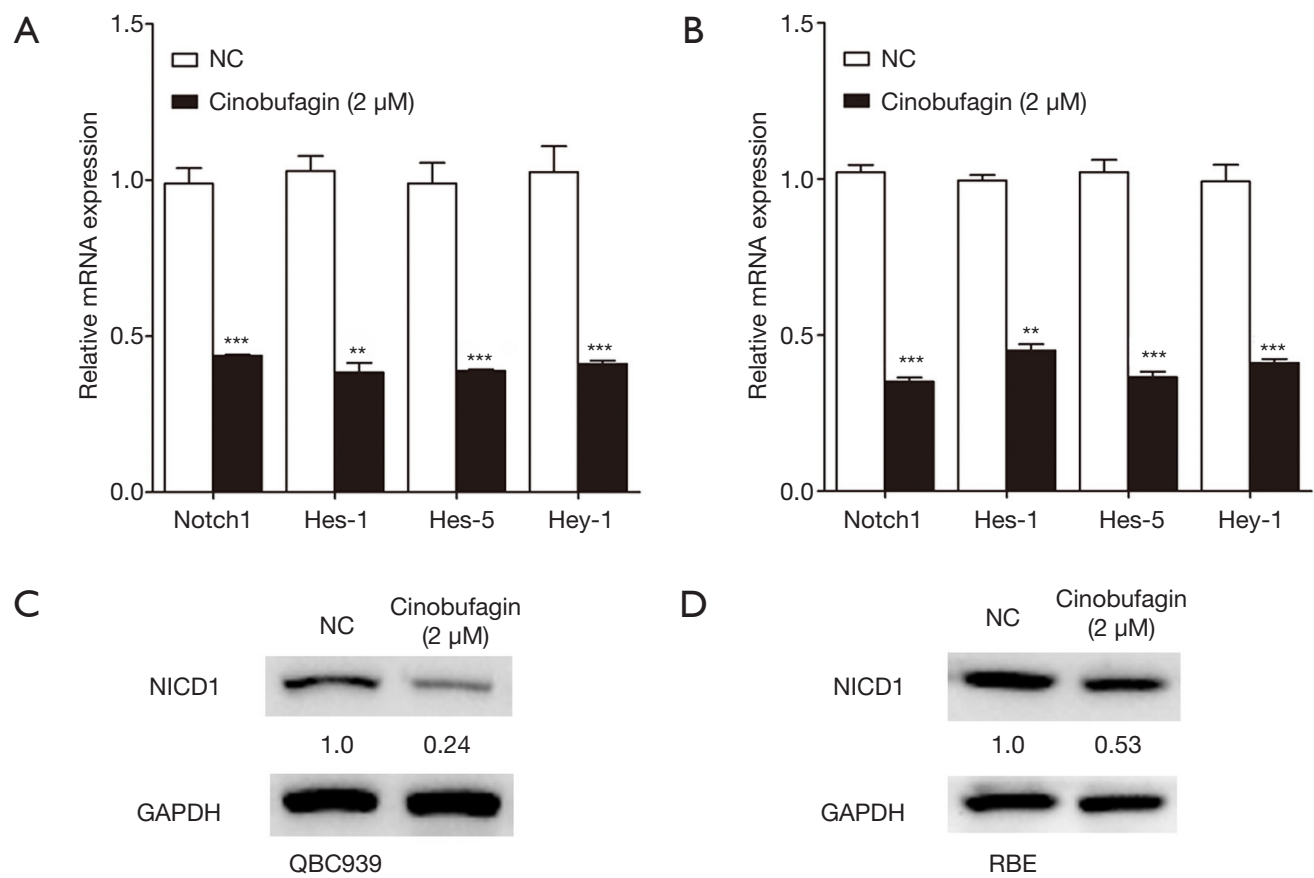

Figure 4 Cinobufagin inactivates Notch 1 signaling pathways. (A,B) The mRNA levels of Notch1, Hes-1, Hes-5, and Hey-1 in human CCA cell line QBC939 and RBE treated with cinobufagin were detected by qPCR; (C,D) protein levels of NICD1 in cell lines QBC939 and RBE treated with cinobufagin were detected by Western blot. ${ }^{* *} \mathrm{P}<0.01,{ }^{* *} \mathrm{P}<0.001$. CCA, cholangiocarcinoma.

including hepatocellular cancer, lung cancer, pancreatic cancer, and colon cancer (12-14,16,22). Experimental studies have suggested that cinobufagin has crucial effects on tumor developing processes including cell proliferation, cell differentiation, apoptosis, cell cycle arrest, angiogenesis, and immune escape $(13,14,16)$.

Previous studies suggested that cordycepin triggered osteosarcoma cell apoptosis via the intrinsic mitochondriadependent apoptosis pathway by the accumulation of ROS and the loss of $\Delta \Psi \mathrm{m}$ (23). In addition, cordycepin has been reported to inhibit breast cancer cell growth and triggers apoptosis by affecting the expression of Bax and Bcl-2 in vitro (15). These are consistent with our present finding that cinobufagin effectively inhibits CCA growth, both in vitro and tumor xenograft experiment in vivo. Furthermore, our mechanistic studies demonstrated that cinobufagin may exert antitumor effects by induction of apoptosis. Notably, it was also found that cinobufagin-mediated inactivation of Notch pathways may play an important role in the induction of apoptosis in CCA, possibly providing a new therapy. In addition, the combination of gemcitabine and cisplatin is the current first-line chemotherapy for patients with advancedstage CCA not amenable to locoregional and surgical options, irrespective of anatomical disease subtype. However, chemotherapy response rate in CCA is low, cinobufagin might be expected to improve the therapeutic effect of these chemotherapeutic agents.

Various signaling pathways could transmit biological information from the outer to inner of cells, however, Notch signaling is the only pathway that conveys the signal into a transcriptional response through cell-cell communication via a ligand-receptor interaction (24). Recent studies have shown that Notch signaling plays an important role in CCA cell proliferation and it contributes to human CCA pathogenesis (25). The Notch signaling pathway is also involved in the genesis and progression of CCA, the activation of Notch plays an important role in bile duct ontogenesis during the steps of ductal plate remodeling and tubulogenesis (26-28). Inhibition of the Notch pathway by $\gamma$-secretase inhibitor IX prevents CCA growth in vitro, and in vivo overexpression of the intracellular domain of Notch together with an inactivation of $\mathrm{p} 53$ significantly increased tumor growth (29). Hence, the inhibition of Notch signaling may have vital therapeutic effects on CCA treatment. Due to the central role of Notch in CCA development and progression, we hypothesized that this pathway is a target 
A

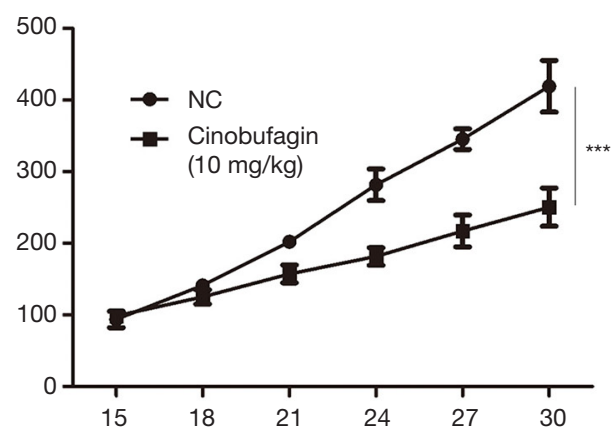

B

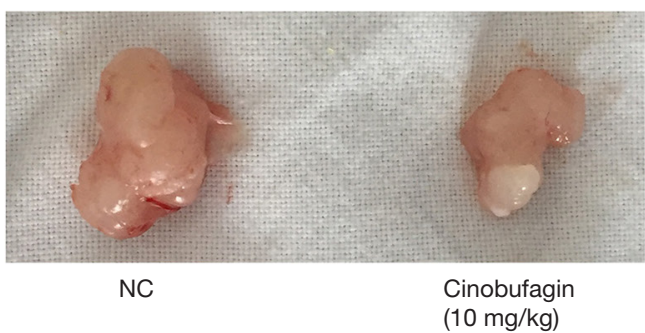

C

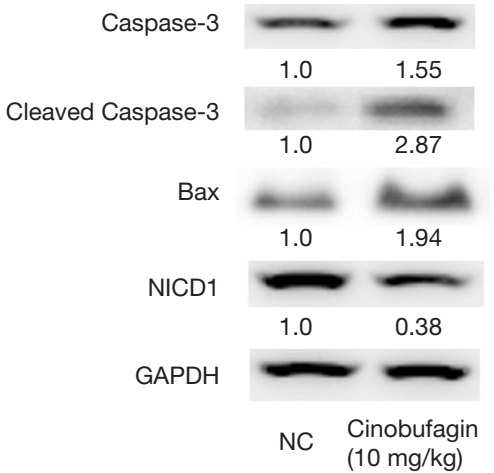

Figure 5 Cinobufagin attenuates growth of CCA in vivo. (A) Growth curves of tumors in nude mice. Tumor diameters were assessed every three days; (B) representative xenograft tumor; (C) protein levels of Caspase-3, Bax, and NICD1 in tumor tissues treated with cinobufagin and detected by Western blot. ${ }^{* *} \mathrm{P}<0.001$. CCA, cholangiocarcinoma.

for cinobufagin. Notch signaling pathway is conserved and responsible for cell-fate determination, differentiation, development, tissue patterning, cell proliferation, and death $(30,31)$. Interaction of Notch ligands with their receptors promotes the release of NICD1 resulting in activation of the pathway. NICD1 translocates to the nucleus and induces target gene transcription like Hes-1, Hes-5, and Hey-1. It has been reported that Notch signaling is frequently overactivated in many cancers, contributing to the survival advantage of tumors (32-35). Thus, Notch activation leads to poorer survival. Because the role of Notch signaling remains tissue and context dependent, alterations within this pathway may lead to tumor suppressive or oncogenic phenotypes. Understanding context-specific effects of Notch pathway will be important for the development of anticancer therapeutics. In the current study, it was found that cinobufagin significantly inactivated Notch signaling pathway.

In conclusion, cinobufagin can suppress the tumorigenicity of CCA, both in vitro and in vivo. Cinobufagin-mediated inactivation of Notch pathway may play an important role in the induction of apoptosis in CCA, possibly providing a new therapy.

\section{Acknowledgments}

Funding: This study is supported by the Interdisciplinary Program of Shanghai Jiao Tong University (ZH2018QNA49).

\section{Footnote}

Conflicts of Interest: The authors have completed the ICMJE uniform disclosure form (available at http://dx.doi. org/10.21037/tcr.2019.10.06). The authors have no conflicts of interest to declare.

Ethical Statement: The authors are accountable for all aspects of the work in ensuring that questions related to the accuracy or integrity of any part of the work are appropriately investigated and resolved. All procedures performed in studies involving human participants were in accordance with the ethical standards of the institutional and/or national research committee(s) and with the Helsinki Declaration (as revised in 2013). The institutional ethical approval and written informed consent were waived. The xenograft study was approved by the Experimental Animal Ethics Committee of Ruijin Hospital.

Open Access Statement: This is an Open Access article distributed in accordance with the Creative Commons Attribution-NonCommercial-NoDerivs 4.0 International License (CC BY-NC-ND 4.0), which permits the noncommercial replication and distribution of the article with the strict proviso that no changes or edits are made and the original work is properly cited (including links to both the formal publication through the relevant DOI and the license). See: https://creativecommons.org/licenses/by-nc-nd/4.0/. 


\section{References}

1. Yang JD, Campion MB, Liu MC, et al. Circulating tumor cells are associated with poor overall survival in patients with cholangiocarcinoma. Hepatology 2016;63:148-58.

2. Udali S, Guarini P, Moruzzi S, et al. Global DNA methylation and hydroxymethylation differ in hepatocellular carcinoma and cholangiocarcinoma and relate to survival rate. Hepatology 2015;62:496-504.

3. Mantel HT, Wiggers JK, Verheij J, et al. Lymph Node Micrometastases are Associated with Worse Survival in Patients with Otherwise Node-Negative Hilar Cholangiocarcinoma. Ann Surg Oncol 2015;22 Suppl 3:S1107-15.

4. Saito H, Noji T, Okamura K, et al. A new prognostic scoring system using factors available preoperatively to predict survival after operative resection of perihilar cholangiocarcinoma. Surgery 2016;159:842-51.

5. Wellner UF, Shen Y, Keck T, et al. The survival outcome and prognostic factors for distal cholangiocarcinoma following surgical resection: a meta-analysis for the 5-year survival. Surg Today 2017;47:271-9.

6. Moeini A, Sia D, Bardeesy N, et al. Molecular Pathogenesis and Targeted Therapies for Intrahepatic Cholangiocarcinoma. Clin Cancer Res 2016;22:291-300.

7. Ye M, Guo H, Guo H, et al. Simultaneous determination of cytotoxic bufadienolides in the Chinese medicine ChanSu by high-performance liquid chromatography coupled with photodiode array and mass spectrometry detections. J Chromatogr B Analyt Technol Biomed Life Sci 2006;838:86-95.

8. Qi J, Tan CK, Hashimi SM, et al. Toad glandular secretions and skin extractions as anti-inflammatory and anticancer agents. Evid Based Complement Alternat Med 2014;2014:312684.

9. Gao H, Zehl M, Leitner A, et al. Comparison of toad venoms from different Bufo species by HPLC and LCDAD-MS/MS. J Ethnopharmacol 2010;131:368-76.

10. Yang Z, Luo H, Wang H, et al. Preparative isolation of bufalin and cinobufagin from Chinese traditional medicine ChanSu. J Chromatogr Sci 2008;46:81-5.

11. Sun Y, Bi J, Zhang L, et al. Ultrasound-assisted extraction of three bufadienolides from Chinese medicine ChanSu. Ultrason Sonochem 2012;19:1150-4.

12. Yu CH, Kan SF, Pu HF, et al. Apoptotic signaling in bufalin- and cinobufagin-treated androgen-dependent and -independent human prostate cancer cells. Cancer Sci 2008;99:2467-76.
13. Qi F, Inagaki Y, Gao B, et al. Bufalin and cinobufagin induce apoptosis of human hepatocellular carcinoma cells via Fas- and mitochondria-mediated pathways. Cancer Sci 2011;102:951-8.

14. Zhang G, Wang C, Sun M, et al. Cinobufagin inhibits tumor growth by inducing intrinsic apoptosis through AKT signaling pathway in human nonsmall cell lung cancer cells. Oncotarget 2016;7:28935-46.

15. Zhu L, Chen Y, Wei C, et al. Anti-proliferative and proapoptotic effects of cinobufagin on human breast cancer MCF-7 cells and its molecular mechanism. Nat Prod Res 2018;32:493-7.

16. Cao Y, Yu L, Dai G, et al. Cinobufagin induces apoptosis of osteosarcoma cells through inactivation of Notch signaling. Eur J Pharmacol 2017;794:77-84.

17. Wang X, Li S, Wan Z, et al. Investigation of thermosensitive amphiphilic micelles as drug carriers for chemotherapy in cholangiocarcinoma in vitro and in vivo. Int J Pharm 2014;463:81-8.

18. Ke F, Wang Z, Song X, et al. Cryptotanshinone induces cell cycle arrest and apoptosis through the JAK2/STAT3 and PI3K/Akt/NFkappaB pathways in cholangiocarcinoma cells. Drug Des Devel Ther 2017;11:1753-66.

19. Rizvi S, Gores GJ. Pathogenesis, diagnosis, and management of cholangiocarcinoma. Gastroenterology 2013;145:1215-29.

20. Padia SA. Intrahepatic Cholangiocarcinoma. Tech Vasc Interv Radiol 2015;18:227-35.

21. Bartella I, Dufour JF. Clinical Diagnosis and Staging of Intrahepatic Cholangiocarcinoma. J Gastrointestin Liver Dis 2015;24:481-9.

22. Lu XS, Qiao YB, Li Y, et al. Preclinical study of cinobufagin as a promising anti-colorectal cancer agent. Oncotarget 2017;8:988-98.

23. Dai G, Zheng D, Guo W, et al. Cinobufagin Induces Apoptosis in Osteosarcoma Cells Via the MitochondriaMediated Apoptotic Pathway. Cell Physiol Biochem 2018;46:1134-47.

24. Cigliano A, Wang J, Chen X, et al. Role of the Notch signaling in cholangiocarcinoma. Expert Opin Ther Targets 2017;21:471-83.

25. Kwon H, Song K, Han C, et al. Epigenetic Silencing of miRNA-34a in Human Cholangiocarcinoma via EZH2 and DNA Methylation: Impact on Regulation of Notch Pathway. Am J Pathol 2017;187:2288-99.

26. Geisler F, Strazzabosco M. Emerging roles of Notch signaling in liver disease. Hepatology 2015;61:382-92.

27. Lemaigre FP. Mechanisms of liver development: concepts 
for understanding liver disorders and design of novel therapies. Gastroenterology 2009;137:62-79.

28. Zong Y, Panikkar A, Xu J, et al. Notch signaling controls liver development by regulating biliary differentiation. Development 2009;136:1727-39.

29. El Khatib M, Bozko P, Palagani V, et al. Activation of Notch signaling is required for cholangiocarcinoma progression and is enhanced by inactivation of $\mathrm{p} 53$ in vivo. PLoS One 2013;8:e77433.

30. Yeh JE, Toniolo PA, Frank DA. Targeting transcription factors: promising new strategies for cancer therapy. Curr Opin Oncol 2013;25:652-8.

31. Capaccione KM, Pine SR. The Notch signaling pathway as a mediator of tumor survival. Carcinogenesis 2013;34:1420-30.

Cite this article as: Ren J, Wang S, Jin L, Ma F, Zhou D, Cai Q. Cinobufagin inhibits tumor growth by inducing apoptosis through Notch signaling pathways in human cholangiocarcinoma. Transl Cancer Res 2019;8(6):2461-2469. doi: 10.21037/tcr.2019.10.06
32. Zhang $\mathrm{H}$, Wang $\mathrm{X}, \mathrm{Xu} \mathrm{J}$, et al. Notch1 activation is a poor prognostic factor in patients with gastric cancer. $\mathrm{Br} \mathrm{J}$ Cancer 2014;110:2283-90.

33. Stoyanova T, Riedinger M, Lin S, et al. Activation of Notch1 synergizes with multiple pathways in promoting castration-resistant prostate cancer. Proc Natl Acad Sci U S A 2016;113:E6457-66.

34. Liu MX, Siu MK, Liu SS, et al. Epigenetic silencing of microRNA-199b-5p is associated with acquired chemoresistance via activation of JAG1-Notch1 signaling in ovarian cancer. Oncotarget 2014;5:944-58.

35. Licciardello MP, Mullner MK, Durnberger G, et al. NOTCH1 activation in breast cancer confers sensitivity to inhibition of SUMOylation. Oncogene 2015;34:3780-90. 


\section{Supplementary}

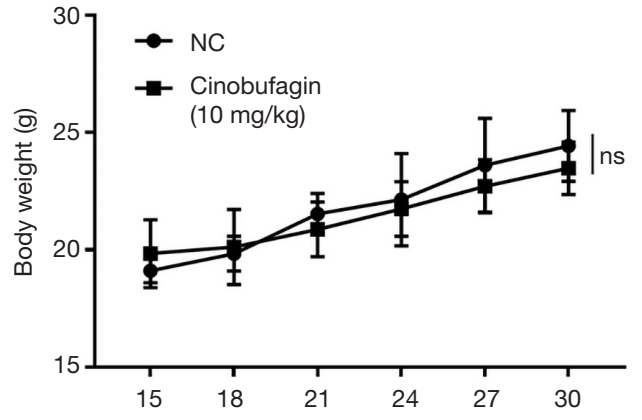

Figure S1 The side effect of cinobufagin is minimal in vivo. Growth curves of body weight of nude mice. Body weight was assessed every three days. Mean \pm SD of triplicate experiments were plotted. ns, not statistically significant. 